\title{
Compaction of Rods: Relaxation and Ordering in Vibrated, Anisotropic Granular Material
}

\author{
Fernando X. Villarruel, Benjamin E. Lauderdale, Daniel M. Mueth, and Heinrich M. Jaeger \\ The James Franck Institute and Department of Physics \\ The University of Chicago \\ 5640 S. Ellis Ave., Chicago, IL 60637
}

\begin{abstract}
We report on experiments to measure the temporal and spatial evolution of packing arrangements of anisotropic, cylindrical granular material, using high-resolution capacitive monitoring. In these experiments, the particle configurations start from an initially disordered, low-packing-fraction state and under vertical vibrations evolve to a dense, highly ordered, nematic state in which the long particle axes align with the vertical tube walls. We find that the orientational ordering process is reflected in a characteristic, steep rise in the local packing fraction. At any given height inside the packing, the ordering is initiated at the container walls and proceeds inward. We explore the evolution of the local as well as the height-averaged packing fraction as a function of vibration parameters and compare our results to relaxation experiments conducted on spherically shaped granular materials.

45.70.-n,05.40.-a,45.70.Cc,81.05.Rm
\end{abstract}

\section{INTRODUCTION}

The packing of identical objects inside a given volume, from atoms to large molecules and polymers to macroscopic particles, is an important problem in many areas of science and technology. For thermal systems, there are well-defined optimal packing configurations, corresponding to thermodynamic equilibrium. In particular, at high packing densities, these equilibrium configurations correspond to ordered, often crystalline, states. However, in many systems, such as glasses, competing interactions between the individual constituents prevent a thermodynamic equilibrium from being reached over experimentally accessible time scales. Furthermore, there are large classes of nonthermal systems, including foams and granular materials such as sand, rice, or pharmaceutical pills, for which ordinary temperature is irrelevant and the usual concept of a corresponding, thermodynamic equilibrium does not exist. In these systems, local temperature-driven fluctuations do not couple to particle motion in an effective manner and do not allow for a full exploration of configuration space. At high packing densities, the most stable, ordered configurations are therefore rarely reached and the packing becomes trapped in metastable, disordered states. Important questions for these strongly non-equilibrium systems remain only partially answered, such as how the metastable configurations respond to perturbations, and how the packing fraction (i.e., the fraction of volume occupied by the granular material) evolves over long times.

Macroscopic granular materials provide a model system for the exploration of these issues [1 3]. In a threedimensional (3-D) packing of monodisperse, rigid spheres held together by gravity and frictional forces, there is a myriad of metastable states, each corresponding to a different packing configuration that satisfies mechanical equilibrium, yet with packing fraction $\rho$ far less dense than the most stable, crystalline configuration for which $\rho \approx 0.74$. Configuration space can be explored conveniently through the application of external mechanical excitations, such as shaking or vibrating. Starting from an initial, low-packing-fraction configuration, the packing evolves over time toward an asymptotic, higherpacking-fraction state with a more compact particle arrangement. For disordered 3-D packings of equal-sized spheres, computer simulations, as well as experiments on steel balls, have found [4, 5 ] that the maximum final packing fraction is set by the random close packing limit, $\rho_{\text {rcp }} \approx 0.64$. Physically, this limit corresponds to amorphous packing configurations that are fully frustrated by geometrical constraints and unable to compact further. We note in this context that a (topological) effective temperature of the packing may be defined in terms of the available free volume [6] or compactivity [7]. Increases in volume fraction then correspond to decreases in this temperature. High densities around $\rho_{\text {rcp }}$ are only reachable via careful cycling of the excitation intensity [8], similar to thermal cycling during annealing procedures. For fixed excitation intensity, the packing will evolve toward final configurations reflecting a balance between defect creation and annihilation. This suggests a second (dynamic) type of effective temperature associated with the strength of the applied forcing. Recent experiments showed that the corresponding final densities $\rho$ are approached logarithmically slow in time [9, 10]. In a number of theoretical models this slow relaxation was explained as arising from geometrical frustration due to excluded volume $\sqrt{10} \sqrt{16}$, and analogies to glassy behavior were drawn [17 20]. Furthermore, it was found that, at short times and low excitation levels, the system can only explore a limited region of configuration space, resulting in highly irreversible behavior and memory effects. Only after sufficiently long times and large excitation levels does one reach a reversible, steady-state response [8,20] 
in the sense that the two effective temperatures track each other, i.e., the packing fraction becomes a monotonic function of applied forcing.

Actual materials, and certainly macroscopic granulates, typically are far from perfectly spherical and often elongated. Under thermal conditions, particle anisotropy is known to give rise to ordering in a variety of systems, such as liquid crystals [21], rod-like colloidal virus particles [22], and certain polymers [23]. For example, a fluid of long, rigid rods at high packing fraction will undergo a transition to an ordered nematic phase in which the rod axes align along a common direction [24]. In nonthermal systems, on the other hand, almost all work to date has focused on spherical particles and the effect of particle anisotropy on the stability and evolution of packing configurations has been largely unexplored. Recent theoretical work by Baulin and Khokhlov [25] on sedimenting solutions of long rigid rods investigated the limit that external (gravitational) forces far outweigh thermal fluctuations and predicts an isotropic to nematic transition for increasing packing fraction. The nature of the transition into the nematic state was studied by Mounfield and Edwards [26] for a model of granular rods. They concluded that an externally imposed (flow) field was required to stabilize a discontinous, first-order-type phase transition into a highly ordered nematic state; otherwise there would merely be a cross-over during which the ordering increases continuously with decreasing compactivity (or increasing packing fraction). Buchalter and Bradley [27,28] used Monte Carlo simulations to study packings of rigid, prolate or oblate ellipsoids. They found that these systems form amorphous, glassy packings with long-range orientational order. Some limited experimental data on the compaction of non-spherical, prolate granular materials under vibrations has been published by Neuman [29], but, to the best of our knowledge, no information on the degree of ordering or on the asymptotically reached, 3-D packing configurations is available. This lack of systematic investigations is surprising, given the enormous importance of prolate granular materials in a wide range of geological and industrial processes.

Here we study 3-D packings comprised of prolate granular material: millimeter-sized rigid cylinders ("rods"). Applying discrete mechanical excitations, or "taps", we let the system evolve from an initial, low-packing-fraction to a final, high-packing-fraction state. During this relaxation process, we monitor the local packing fraction noninvasively and correlate it with direct visual images of the packing configurations. We are specifically interested in the question of how two competing factors affect the packings' evolution as the packing fraction increases: on the one hand, the tendency of randomly arranged rods to lock up in a disordered state because of steric hindrance and friction, and on the other hand the possibility, provided by both gravity and container walls, to align and form ordered, nematic-type configurations. Our results show that there are characteristic stages to the evolution, corresponding to either process. We find that, depend- ing on the tapping history and intensity, highly ordered final states are achievable, in contrast to sphere packings under the same experimental conditions.

This paper is organized as follows. In Section II we describe the experimental set-up and procedure. Results on the relaxation and alignment behavior for a range of excitation intensities are shown in Section III and discussed in Section IV. Section V contains a summary of the findings and conclusions.

\section{EXPERIMENTAL SET-UP AND PROCEDURE}

All experiments were performed on monodisperse nylon $6 / 6$ rods of specific density $(1.145 \pm 0.005) \mathrm{g} / \mathrm{cm}^{3}$, each $1.8 \mathrm{~mm}$ in diameter and $7.0 \mathrm{~mm}$ in length. Approximately 7200 of these rods were filled into a $1 \mathrm{~m}$ tall glass tube $(1.90 \mathrm{~cm}$ inner diameter $)$ mounted vertically on a Bruel and Kjaer 4808 electromagnetic vibration exciter (Fig. 1a). As in previous experiments on spherical particles 810,30 , vertical vibrations were applied in the form of individual shaking events ("taps") by driving the exciter with a single cycle of a $30 \mathrm{~Hz}$ sine wave. Successive taps were spaced by time intervals sufficiently long (typically $0.5 \mathrm{~s}$ ) to allow complete relaxation of the system. The vibration intensity was monitored using an accelerometer. In the following we parameterize the tapping intensity by $\Gamma$, the ratio of the measured peak acceleration to the Earth's acceleration $\mathrm{g}=9.81 \mathrm{~m} / \mathrm{s}^{2}$ (Fig. 1c). The bottom of the tube contained an entry way for dry nitrogen, which was used only in the preparation of the initial, low packing fraction state of the sample. Through the top of the glass tube the system was placed under vacuum during runs. A control tube similar to the tube described above, filled with the same material but not vibrated, was used to measure electronic drift.

The evolution of the packing fraction between taps was monitored both globally by recording the total filling height of material inside the tube, and locally using a capacitive technique. Mounted along the outside of the tube were four capacitors made from pairs of copper strips, each $1.25 \mathrm{~cm}$ wide and $17 \mathrm{~cm}$ in length. Each of the capacitors was sensitive to a measurement volume inside the tube defined by a slab of cross-sectional area as indicated in Fig. 1b (about $70 \%$ of the total crosssectional area of the tube). We ascertained that there was little sensitivity to material placed outside this active volume. The capacitance was read by a capacitance bridge with $1 \mathrm{fF}$ resolution. The relation between packing fraction, $\rho$, and capacitance, $C$, was found to be linear, $\rho=-3.47 \times 10^{-1}+2.35 \times 10^{-3} C$, where $C$ is measured in $\mathrm{fF}$ (Fig. 2). The data in this figure contains data from all capacitors, with readings for the empty $(\rho=0)$ tube and for a solid nylon rod occupying its total volume $(\rho=1)$, as well as data for intermediate packing fractions. For the latter, the two methods employed, a) inserting a solid rod 

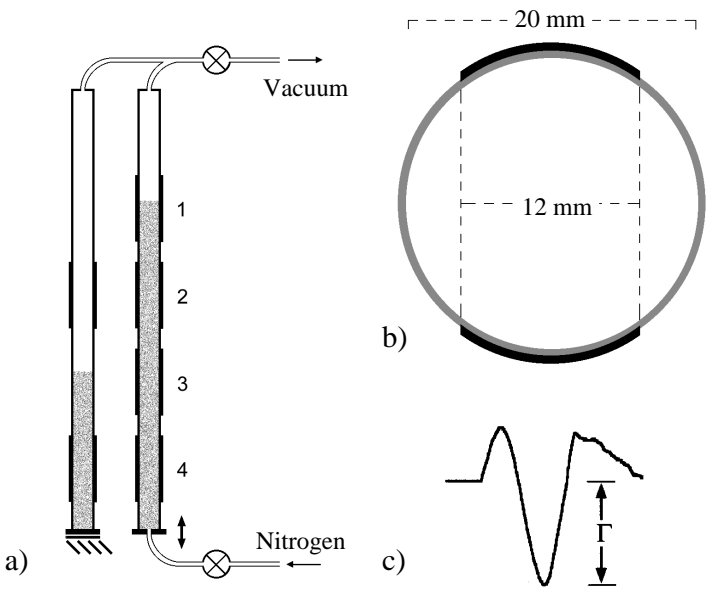

FIG. 1. (a) Schematic of the experimental system used for capacitive measurements of the packing fraction. The set-up consists of a measurement column with four capacitors and a control column with two capacitors for tracking electronic drift. The initial packing state is prepared by fluffing with dry nitrogen injected through the valve shown at the bottom. (b) Sketch of the measurement tube cross-section. Two capacitor electrodes are shown at the tube perimeter. The active region sensed by the capacitor lies between the dashed lines. (c) Time trace of an individual, vertical "tap" as measured by the accelerometer. The peak acceleration, $\Gamma$, is indicated.

partially into a capacitor or b) compacting a test sample of cylinders with a known number of particles inside the measurement volume, both yielded the same results, indicating that particle shape effects do not significantly influence the packing fraction measurements.

Because visual tracking of particles through the tube side walls was limited to areas between capacitors, we used a separate set-up with a tube of same diameter but shorter $(21 \mathrm{~cm})$ and without capacitors to explore qualitatively the evolution of particle orientations. This was done by video-taping the material through the outside walls and also from above. In addition, careful layer-bylayer vacuuming allowed us to map out the depth dependence.

For each run, the $1 \mathrm{~m}$ tall tube was filled with $143.1 \mathrm{~g}$ of material to a height of $(82.3 \pm 0.3) \mathrm{cm}$. The material was then fluffed with nitrogen to an initial filling height of $(90.6 \pm 0.5) \mathrm{cm}$, corresponding to a packing fraction $\rho=0.49$. We found this to represent the least dense, reproducible initial packing state attainable. Both measurement and control tube were then placed under vacuum to isolate them from environmental changes in the room during a run.

Here we describe two sets of experiments. In the first set, the material was tapped 70,000 times at fixed acceleration and capacitance readings were made after certain, fixed tap intervals. Simultaneously, the average packing fraction of the column was recorded by measuring the overall filling height with a ruler. Prior to each experi-

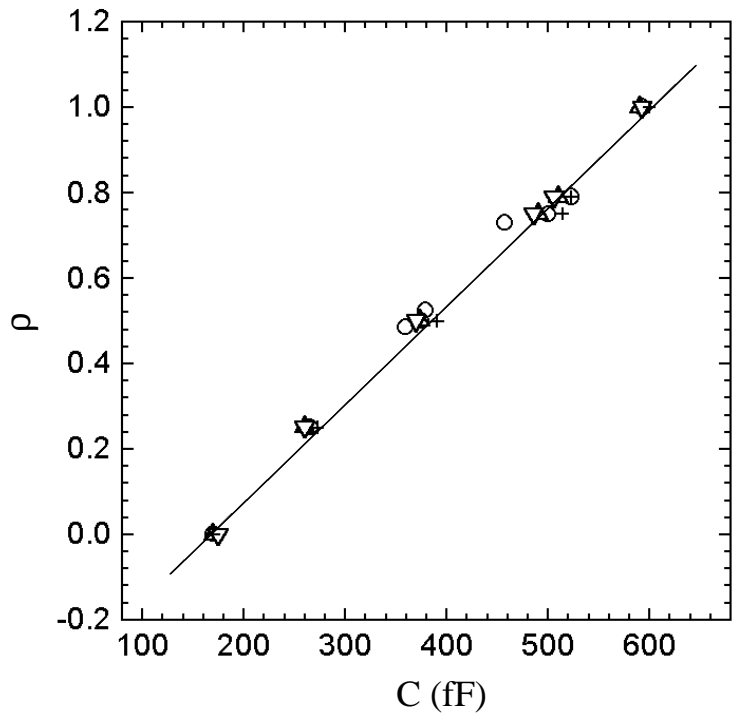

FIG. 2. The capacitor calibration used to determine the packing fraction, $\rho$, from the measured capacitance, $C$, at various heights in the column. The circles, triangles, down triangles, and crosses are for capacitors $1,2,3$, and 4 , respectively. The data is seen to be well-fit by a linear relationship.

mental run, the system was returned to the same loosepacked initial state by removing all material, refilling and fluffing with nitrogen. This was done to remove all traces of ordering from previous runs. In the second set of experiments, we explored the effect of tapping history on the packing fraction evolution. A fixed number, $\Delta t$, of taps were applied to the system and the final packing fraction was recorded. Without refilling or fluffing the system, the acceleration was adjusted by $\Delta \Gamma$ and the measurement process was continued, ramping $\Gamma$ from 1.5 to 7.5 and back several times. This is similar to what would be done in a cyclic heating and cooling process, with $\Delta \Gamma / \Delta t$ playing the role of an effective heating or cooling rate.

\section{RESULTS}

Figure 3 shows the evolution of the packing fraction, $\rho(t)$, for different capacitor regions as a function of tap number, $t$. Data for four different accelerations are shown. Each curve is an average of five independent runs and the error bars, for sake of clarity given only for capacitor 2 in the $\Gamma=4.5$ graph, represent the general rms variation. In order to be able to display the initial packing fraction, $\rho(0)$, the tap number (or time) axis on all plots has been incremented by one tap.

The evolution shown in Figure 3 exhibits three distinct stages which we call the initial relaxation stage (up to about $10^{3}$ taps), the vertical ordering stage (between roughly $10^{3}$ and $10^{4}$ taps), and the final, steady-state regime. 


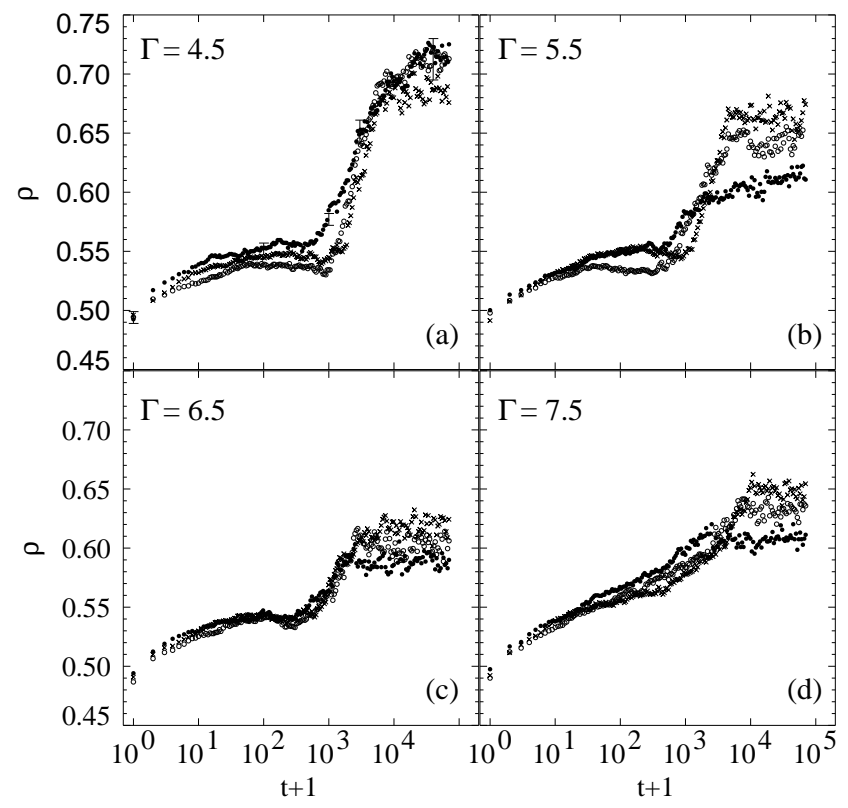

FIG. 3. The evolution of the packing fraction, $\rho(t)$, as a function of tap number, $t$, for several accelerations, $\Gamma$, as indicated. Each plot contains traces for regions 2, 3, and 4 along the height of the tube (Fig. 1a), shown by solid circles, open circles, and crosses respectively (we excluded region 1 since it was partially emptied during the compaction of the column). Most pronounced for smaller $\Gamma$, we observe three distinct regimes, corresponding to separate physical processes as explained in the text.

During the initial relaxation stage there is a quick increase in $\rho$ during the first decade and then a leveling off to a plateau near 0.55 . This saturation is highly pronounced for $\Gamma=4.5,5.5$ and 6.5 , but much less so for $\Gamma=7.5$. The second stage is identified by an abrupt increase in packing fraction, which becomes less steep and smaller as $\Gamma$ increases. This increase in packing fraction coincides with a nematic ordering of the material, during which the material aligns vertically parallel to the tube walls. Figure 4 shows snapshots of particle configurations during this alignment process, taken midway down the height of the tube for $\Gamma=4.5$ : (a) and (b) are side views, as seen through the tube wall, of the initial, randomly packed state, and the highly aligned arrangement of the outer layer at some point toward the end of the ordering stage, respectively.

Images (c)-(f) show top views of the packing interior, obtained after careful vacuuming out the material in the upper half of the column. The initial state (c) shows the material as poured (but not fluffed). After 2000 taps (d), the particles have lined up along the edge of the tube, while its interior remains disordered (start of ramping domain). After 6000 taps (e), the particles in the interior also have begun to orient vertically. The final, steady-state regime is a dense, highly aligned configuration (f). This image sequence demonstrates that the vertical alignment starts at the tube walls and proceeds

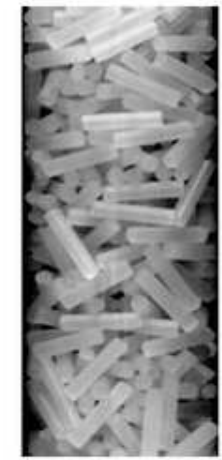

(a)

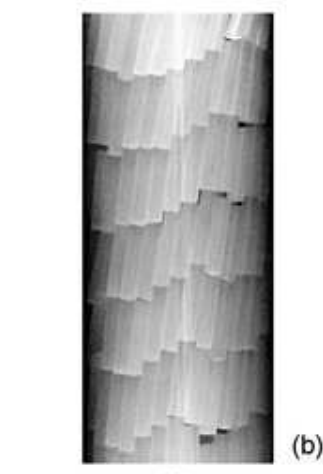

(b)

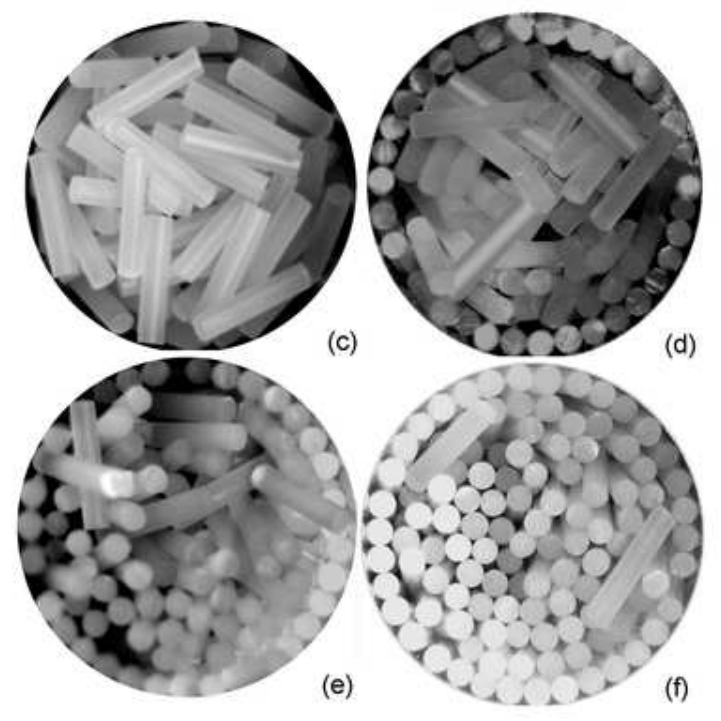

FIG. 4. Snapshots of particle configurations at various times during the packing evolution. (a) The particle configuration, as viewed from the side, is disordered after the material is initially filled into the column. (b) After relaxation caused by many thousands of taps the particles line up vertically and can form a highly ordered, nematic state. The progression from disordered to ordered is best seen by carefully removing the material in the upper half of the column and viewing from above (c-f). The initial, disordered state (c), evolves into a state with order only along the wall (d) after about 2000 taps at $\Gamma=4.5$. After about 6000 taps, order has propagated in from the walls (e), although the particles are not yet perfectly aligned with the vertical in a nematic state. The long time behavior (f) is a nematic state with a $\Gamma$-dependent degree of disorder in the interior, similar to that viewed from the side in (b). 
inward. We find that the start of the ordering stage varies slightly with height along the tube, for $\Gamma<5$ happening somewhat earlier at the top of the tube and moving downward (see Fig. 3a). At higher accelerations, $\Gamma=6.5$ and 7.5, the vertical alignment at the end of the ordering stage is less perfect and at the highest accelerations explored, $\Gamma=7.5$, the ordering stage becomes less distinct, particularly for the lower regions of the container. Final, steady-state configurations at these accelerations are very similar to those in Figure 4e, where only the outside is well aligned while the inside still exhibits numerous defects.

In the final steady-state regime both the initial relaxation and subsequent vertical ordering have saturated and the packing fraction is found to fluctuate around a constant, asymptotic value (Fig. 3). This final packing fraction typically increases with depth below the free surface (in our data for $\Gamma=4.5$ the differences may be too small to be significant). For accelerations below $\Gamma=4.5$ we found the dynamics to be exceedingly slow, preventing an asymptotic state from being reached by $t=10^{5}$, the longest tapping interval explored in these experiments.

In Fig. 5 we show the average overall packing fraction as determined by the total height of the material in the tube, $\rho_{h}(t)$. Clearly visible is the significant increase of final packing fraction for $\Gamma=5.5$ and below. In general, $\rho_{h}(t)$ mimics the three stages seen in $\rho(t)$. However, the onset of the vertical ordering regime is not as abrupt for $\rho_{h}(t)$ as it is for $\rho(t)$, nor is it preceded by the slight dip in volume fraction which ends the first domain in Figure 3. A direct comparison of $\rho_{h}(t)$ with the height-averaged packing fraction, $\left\langle\rho(t)>_{h}\right.$, obtained from the capacitor data in Fig. 3, is shown in Fig. 6. As we will discuss below, the differences between the two types of measurement reflect the fact that the active volume responsible for $\rho(t)$ includes only a fraction of the material near the tube wall. Also included in Figs. 5 and 6 is a trace for $\Gamma=4.5$ obtained under different initial conditions: instead of initial fluffing, the material was merely dropped into the tube and then tapped. We note that the memory of the preparation conditions persists only up to about 20 taps, after which traces for different initial conditions coincide.

Results from the second type of experiment are given in Fig. 7 for $\Delta t=100$ (a) and 1,000 (b), using $\Delta \Gamma=1.0$ in both cases. The ramp rates are fast enough that the material does not have time to reach the steady-state during the first pass. Consequently, the packing fraction initially does not depend on acceleration alone, but also strongly on the vibration history: As $\Gamma$ is ramped first up, and then down and up again several times, $\rho_{h}$ slowly cycles toward a reversible regime in which $\rho_{h}(\Gamma)$ becomes monotonic. For fast rates, as in Fig. 7a, a large number of cycles may be required; for slower ramp rates the reversible regime may be approached much earlier (Fig. 7b).

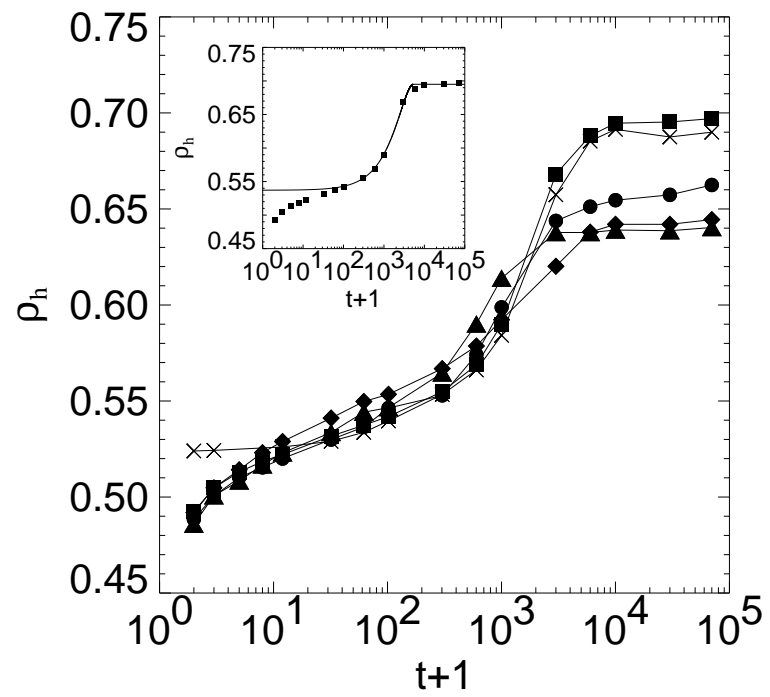

FIG. 5. The time evolution of packing fraction as determined by column height, $\rho_{h}(t)$, for shaking intensities $\Gamma=4.5,5.5,6.5$, and 7.5 , represented by squares, circles, triangles, and diamonds, respectively. The three stages seen in the capacitance data in Fig. 3 are also visible here. The crosses represent $\rho_{h}(t)$ for an experiment with different initial conditions in which the fluffing step in the system preparation was omitted. The inset shows a fit of the packing fraction for $\Gamma=4.5$ throughout the vertical ordering regime to $\frac{\rho_{h}(t)-\rho_{h}\left(t_{i}\right)}{\rho_{h}\left(t_{f}\right)-\rho_{h}\left(t_{i}\right)}=2 \frac{t-t_{i}}{t_{f}-t_{i}}-\left(\frac{t-t_{i}}{t_{f}-t_{i}}\right)^{2}$, where $t_{i}$ and $t_{f}$ are the beginning and ending time of the vertical ordering stage, respectively, as discussed in Section IV.

\section{DISCUSSION}

Two central observations from recent, systematic compaction experiments 8 10,30] with spheres were a) at fixed acceleration the logarithmically slow approach in time toward the final steady-state packing fraction, and b) the existence of memory effects in which the final state can depend on the initial sample preparation and on the acceleration history. In particular, from analysis of the packing fraction fluctuation spectra it was found that there is an intrinsic, broad range of relaxation time scales, many of which, however, are effectively "frozen out" if the acceleration stays below certain values [10,20].

Much of this behavior qualitatively carries over to the cylinder-shape particles investigated here. The data in Figs. 3 and 6 indicate a non-exponential relaxation of voids in the packing during the first stage of the packing fraction evolution. At the highest acceleration, when effects due to particle alignment are weakest (Figs. 3d and $6 \mathrm{~d})$, we find that $\rho(t)$ increases approximately logarithmically before it eventually saturates. Such logarithmic relaxation arises naturally from free volume considerations, in which the ability of particles to find and occupy any of the remaining free space decreases exponentially in time 10,11,16. In its simplest form, this scenario is independent of particle shape and, furthermore, only 


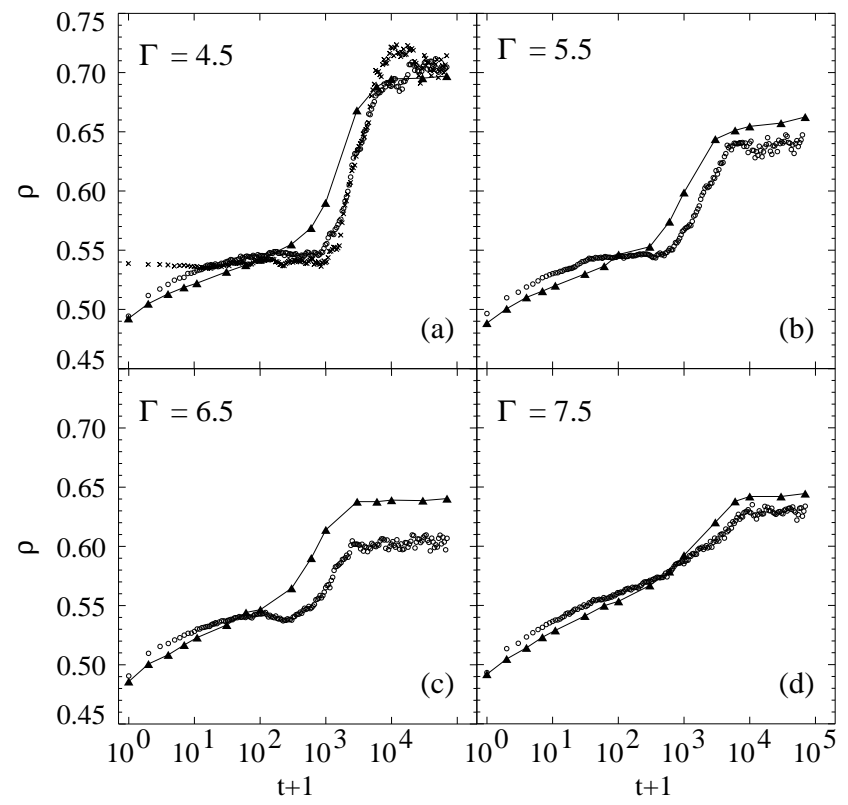

FIG. 6. Comparison of packing fraction evolution as determined by height and capacitance. The packing fraction as determined by height, $\rho_{h}(t)$, is shown as solid triangles. The average packing fraction of the column, $\langle\rho(t)\rangle_{h}$, determined by averaging the capacitive measurements in Fig. 3, is shown as open circles. The difference between the curves reveals the existence of a radial packing fraction profile which evolves over time as the system orders. The crosses shown in (a) represent $\langle\rho(t)\rangle_{h}$ for different initial conditions in which the fluffing step in the system preparation was omitted.

based on void annihilation. Without the additional possibility of defect or void creation, however, the system eventually has to jam at a final packing fraction that can only be a monotonically increasing function of applied acceleration (since hindrance effects are more effectively overcome at larger $\Gamma$ ). Instead we find that, over the range in $\Gamma$ explored, the overall final packing fraction decreases with increasing acceleration (Figs. 5, 6). As for spheres, this clearly indicates defect production in response to tapping. Nevertheless, as Fig. 7 shows, memory of the tapping history is not easily destroyed and can persist over ten-thousands of taps: if during this time the tapping intensity is changed, the system irreversibly moves into a new state of higher packing fraction, independent of whether the tapping intensity is increased or decreased [31]. Only at sufficiently high acceleration and after sufficiently many taps is the reversible regime reached where the packing fraction depends monotonically on acceleration. This situation is similar to superheating or super-cooling in thermal systems.

The prolate, anisotropic particle shape amplifies the ability of 3-D cylinder packings to sustain large voids. This is demonstrated by the low packing fraction $(\rho(0)=$ 0.49 in Fig. 3 compared to 0.59 for spheres in the same experimental set-up [9]) for the loosest, mechanically stable configuration, by the large overall compaction range
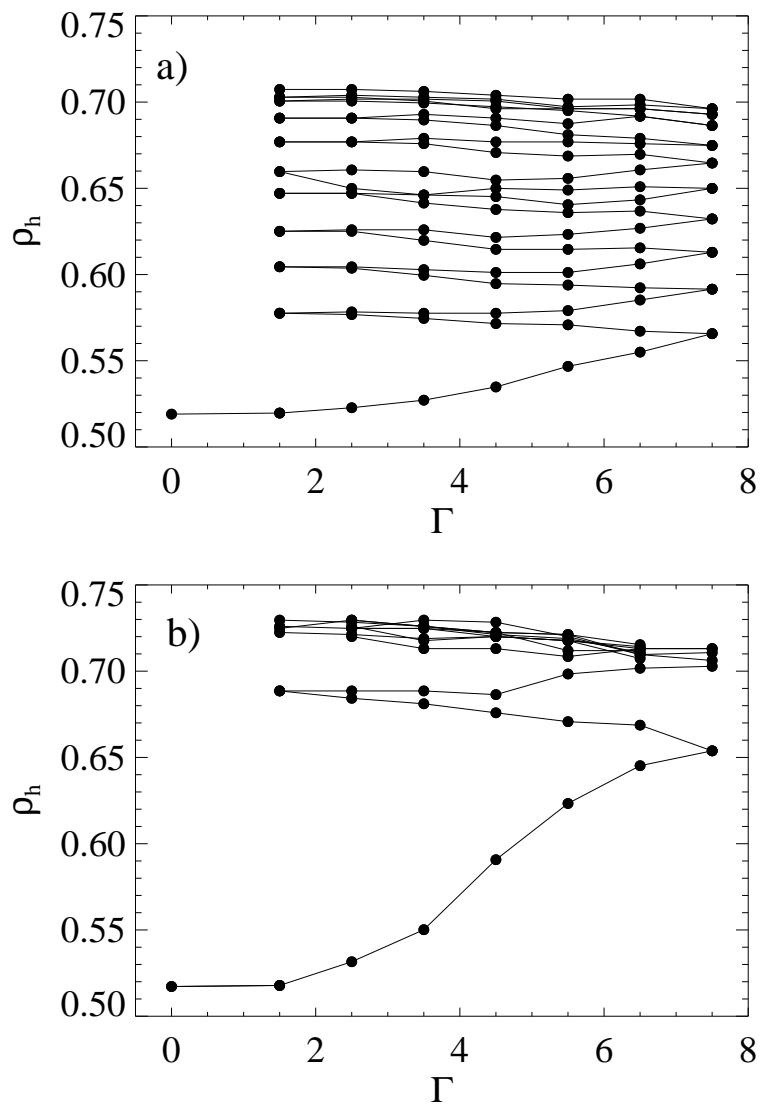

FIG. 7. Packing fraction, $\rho_{h}$, at various stages during experiments in which the system was not allowed to reach a steady-state before changing the acceleration $\Gamma$. In (a), the system was tapped for $\Delta t=100$ taps at each acceleration as $\Gamma$ was ramped from 1.5 to 7.5 and back for several cycles in steps of $\Delta \Gamma=1.0$. The system increases in packing fraction irreversibly before reaching a reversible steady-state branch where $\rho$ becomes a monotonic function of $\Gamma$. In (b) the time interval was increased to $\Delta t=1000$, allowing the system to relax more at each acceleration value. The reversible branch is reached after only 1.5 cycles.

$(0.49<\rho<0.72$ in Fig. 3a compared to $0.59<\rho<0.65$ for spheres [9]), and by the large fluctuations in $\rho(t)$ in the steady-state.

As Fig. 7b shows, packing defects can be annealed out, first by either "heating" or "cooling" along an irreversible branch and, once the reversible branch is reached, by "cooling" to final packing fractions as high as 0.73 . We note that this value is roughly $10 \%$ smaller than the random close packing density in two dimensions [32], $\rho_{\mathrm{rcp}}^{2 \mathrm{D}}=0.82$, defined as the maximum packing fraction beyond which a transition to an ordered triangular array would be necessary. Along the reversible branch, memory of the acceleration history is erased as long as "heating" or "cooling" steps are taken at sufficiently slow rates $\Delta \Gamma / \Delta t$. However, the maximum allowable rate itself depends on how far the system has evolved: With $\Delta \Gamma / \Delta t=10^{-2}$, in Fig. $7 \mathrm{a}$, the system clearly was cy- 
cled too fast ("superheated" as well as "supercooled") and stayed in the irreversible regime even at the highest $\Gamma$ until it had time to relax. But, once the steady-state is reached, the same fast rate produces very little, if any, "supercooling".

Qualitatively, the response to acceleration cycling seen in Fig. 7 is similar to that obtained for spherical particles [8]. Presumably, therefore, concepts based on frustration due to volume exclusion alone may model the observed behavior. However, the shape of $\rho(t)$ differs dramatically from the sphere case because of the vertical ordering regime. Presently available models [7, $11,20,33$, 35] for $\rho(t)$ do not account for the orientational degree of freedom and thus are not applicable without modification.

The most crucial difference between sphere and cylinder packings comes from the tendency of cylinders to align along their long axis, both with each other and with the container walls. Vertical alignment along the direction of the tube walls becomes noticeable after initial void relaxation has started to saturate (typically after $\approx 100$ taps in Figs. 3 and 6 ). After the void relaxation process saturates, vertical particle ordering becomes the dominant mechanism for compaction. Note that, over the acceleration range explored, the material once vertically aligned is highly stable against reorientation.

This ordering process bears resemblance [26] to the transition from isotropic to nematic phases in liquid crystals [21] and for hard rods in thermal systems [24]. As in a nematic, particle motion in the aligned granular state is found to occur mainly by translation parallel to the cylinder axis. In cases where the aligned system was particularly carefully cooled, we also observed vertical stacking (seen for the outermost particle layer in Fig. 4b) akin to smectic-type phases in liquid crystals.

As seen from Fig. 4, at any given height inside the tube, the first particles to vertically align are those in contact with the tube walls. Alignment then progresses horizontally inward, until the whole tube cross-section is ordered. This progression is also detected non-invasively from the difference in the packing fraction values obtained by filling height $\left(\rho_{h}(t)\right)$ and by capacitance $\left(\rho(t),\langle\rho(t)\rangle_{h}\right)$. The fraction of particles near the tube walls covered by the active measurement volume of the capacitors is less than $50 \%$ (Fig. 1b), making the capacitive measurements more indicative of the packing fraction in the central tube region, along its axis. For this reason, $\langle\rho(t)\rangle_{h}$ is less than $\rho_{h}(t)$ during the vertical ordering stage until the ordering front has reached the tube center and the final steady state is obtained (Fig. 6).

In the simplest possible picture, the increase in packing fraction during the ordering stage is solely due to conversion of disordered three-dimensional particle arrangements with large void space to a dense, highly aligned configuration of essentially two-dimensional character. A model of this process can be constructed as follows. We assume that cylinders in the disordered state can align vertically only in the presence of previously aligned neighbors that act as nucleation sites (this assumption of an essentially step-like front separating the isotropic from the nematic regions is supported by the calculations of Baulin and Khokhlov [25]). Then the ordering proceeds at a rate $\partial N / \partial t=\mathrm{p} n$, where $N$ is the total number of already lined-up cylinders behind the ordering front, $n$ is the number of open nucleation sites and $\mathrm{p}$ a fixed probability for alignment. Through the two-dimensional packing fraction for discs, $N$ and $n$ are related to the size of the ordered area and its inner perimeter, respectively. For a cylindrical tube we find, $n \propto \sqrt{N_{f}-N}$, where $N_{f}$ is the final number of aligned particles at $t=t_{f}$, the end of the ordering stage. The square root dependence reflects the fact that the number of nucleation sites shrinks as the ordering front advances radially from the tube wall toward the tube center (the tube wall acts as a ring of nucleation sites at $t=t_{i}$ when $N\left(t_{i}\right)=0$ ). As a result, the fraction of ordered cylinders increases with tap number as $\frac{N(\tau)}{N_{f}}=2 \tau-\tau^{2}$ for $0 \leq \tau \leq 1$, where $\tau=\frac{t-t_{i}}{t_{f}-t_{i}}$ is the normalized tap number. The measured increase in packing fraction is then directly proportional to $N(\tau) / N_{f}$. If we take as the start of the ordering stage $\left(t=t_{i}\right.$ in the above expression) the point in time at which $\langle\rho\rangle_{h}$ first falls below $\rho_{h}$, we find that the above functional form for $N(\tau)$ fits the data quite well (inset to Fig. 5).

Radial packing fraction gradients, detected by differences between $\langle\rho(t)\rangle_{h}$ and $\rho_{h}(t)$, are also found in the initial relaxation stage and in the final steady-state regime. During void relaxation, the capacitive data in Fig. 6 typically lie above those from the height measurements, indicating a slightly higher packing fraction in the central region, away from the walls. This is a consequence of two effects. First, the tube wall can provide stable pinning, preventing low-packing-fraction configurations from collapse (initial void collapse is accelerated if the material is merely dropped into the column, rather than carefully fluffed, as seen in Fig. 6a). Second, next to the tube wall, the packing fraction naturally is reduced due to excluded volume (unless the packing configuration is commensurable with the tube volume). Of course, once alignment along the tube wall has begun, the outer regions become denser than the tube interior and the traces for $\langle\rho(t)\rangle_{h}$ and $\rho_{h}(t)$ cross. The slight dip present in $\rho(t)$ or $\langle\rho(t)\rangle_{h}$ at the end of void relaxation and the beginning of the ordering stages (for $\Gamma \leq 6.5$ ) indicates that the packing fraction temporarily decreases in the central tube region. We speculate that this local dilation might be required to allow particles to rotate and align with the tube wall.

At high accelerations, $\Gamma \geq 5.5$, a radial gradient in the packing fraction remains even in the steady-state regime (Fig. 6). At these accelerations, particles near the wall remain highly aligned while the interior exhibits packing defects (similar to Figs. 4d, e). Consequently, the capacitively measured steady-state packing fraction values fall below those from the height measurements (Fig. 6b-d). Interestingly, for $\Gamma=7.5$ this gradient develops only at large times. Conversely, for $\Gamma=4.5$ the fact that $\langle\rho(t)\rangle_{h}$ 
and $\rho_{h}(t)$ coincide at large times indicates a highly uniform packing fraction profile across the tube once the asymptotic state is reached (cf. Fig. 4e).

As far as particle configurations are concerned, the simulations of prolate ellipsoid packings by Buchalter and Bradley [27,28] predict that the pouring and packing process by itself should lead to a certain degree of nematic ordering ("nematic glass"). In a (infinite) system without vertical side walls this would be a consequence of minimizing the gravitational potential energy during particle deposition: the first rods hitting the container bottom would tend to lay flat and thus induce horizontal alignment of subsequent layers. Such a state contains fewer large voids than a completely isotropic packing configuration and is thus denser. In our experiments, this might be reflected in the difference between the as poured and fluffed traces for $\Gamma=4.5$ in Figs. 5 and 6 a. Indeed, as the side and top views in Figs. 4a and c show for the poured initial state, there is a preference for cylinders to orient toward the horizontal. As the eventual merging of the $\rho(t)$ curves for different initial conditions indicates, the same vertically "flattened" packing state is also reached from the more isotropic, fluffed initial condition, namely as most large voids have collapsed and $\rho(t)$ starts to level off (Fig. 6a). At least for accelerations $\Gamma \leq 6.5$ this packing state can be identified with a packing fraction $\rho \approx 0.55$

Our present system is too small (in terms of lateral extent) to cleanly test whether the packing configuration at the end of the void relaxation stage corresponds to a horizontally orientated "nematic glass" state. According to Ref. 27] shaking is expected to eventually break up this orientational order and our data are certainly compatible with this for long times and/or high accelerations. However, vertical shaking in the presence of the tube sidewalls, rather than merely reducing the degree of horizontal ordering, provides a strong incentive for rods to line up vertically, similar to the situation in a thermal system of rods where the loss in rotational entropy is compensated by a gain in free volume accessible to translations. (As we mentioned above, this gain may be the cause of the small dip in the packing fraction that we pick up by the capacitive measurements in Figs. 3ac before the vertical ordering changes the overall particle packing fraction.) In principle, this argument might also lead to some initial vertical ordering along the tube walls from the pouring process. From Figs. 4a and c, and also from the fact that the initial densities $\langle\rho(0)\rangle_{h}$ and $\rho_{h}(0)$ coincide in Fig. 6, we find, however, no evidence of such alignment, demonstrating that gravitational potential energy far outweighs rotational entropy unless it is mitigated by the applied acceleration during tapping.

\section{CONCLUSIONS}

We have extended our investigations of the relaxation behavior of nonthermal, granular material to highly anisotropic, cylindrical particle shapes. Using a combination of non-invasive, capacitive probes and video imaging, we have traced both the local and global packing fraction $\rho$ as well as the evolution of the packing configurations (Fig. 4) from an initial, low-packing-fraction to a final, high-packing-fraction state under applied mechanical excitations.

We observe many qualitative features also seen in the relaxation of sphere packings and find them amplified by the particles' anisotropy. This includes an even wider range of metastable configurations and thus a larger span of accessible packing fractions (Fig. 3), as well as memory effects in the irreversible branch of $\rho(\Gamma)$ that are much more pronounced (Fig. 7).

In contrast to sphere packings, which tend to end up in disordered configurations even after prolonged tapping and cycling, we find clear evidence that particle anisotropy can drive ordering. This is most strikingly observed in $\rho(t)$ where we can distinguish three characteristic stages (Figs. 3 and 6): First, a void relaxation stage takes the initially isotropic arrangement to an intermediate packing fraction near $\rho=0.55$. This is a state of vertically collapsed and thus predominantly horizontally oriented particle configurations which may correspond to the nematic glass state seen in computer simulations 28 . This state, however, turns out to be unstable to continued vertical excitations. During a second stage, the vertical ordering regime, particles re-align their long axes vertically. This ordering process starts from the container walls, moves inward and eventually leads to an ordered, nematic-type configuration. We have shown that a simple model can account for the change in packing fraction during this ordering process. The imposed boundary conditions at the wall stabilize the nematic state, effectively playing a role similar to strong flow fields [26] or packing fraction gradients [25]. The third, and final, stage is a steady-state with large fluctuations around an average packing fraction set by competing defect annihilation and creation within the nematic-type particle arrangement.

These results provide a first experimental step toward the full exploration of the effect of anisotropy on ordering in strongly non-equilibrium, nonthermal systems. Our experiments used a fixed aspect ration of close to four. Larger aspect ratios as well as less rigid particles are expected to hinder the transition from isotropic to nematic configurations, and there is also evidence that oblate particles should order differently from the prolate ones investigated here 28]. Another intriguing extension concerns mixtures of spheres and rods. For a thermal system of this type, novel micro-phase separated patterns, such as lamellae of spheres and rods, have recently been found experimentally [36], while theoretical models for the granular equivalent would suggest macroscopic phase separa- 
tion [37. Finally, for none of the non-spherical systems has the spectrum of fluctuations around the steady-state been explored yet.

\section{ACKNOWLEDGMENTS}

We wish to thank Damien Dawson, Allan Smith, Tom Witten and, in particular, Sidney Nagel for many helpful and illuminating discussions. This work was supported by the NSF under Award CTS-9710991 and by the MRSEC Program of the NSF under Award DMR-9808595.

[1] A. Mehta, Granular Matter, An Interdisciplinary Approach (Springer Verlag, New York, 1994).

[2] P.-G. de Gennes, Reviews of Modern Physics 71, S374 (1999).

[3] H. M. Jaeger, S. R. Nagel, and R. P. Behringer, Review of Modern Physics 68, 1259 (1996).

[4] J. L. Finney, Proc. Roy. Soc. Lond. A 319, 479 (1970).

[5] K. Z. Y. Yen and T. K. Chaki, Journal of Applied Physics 71, 3164 (1992).

[6] M. Shahinpoor, Powder Technology 25, 163 (1980).

[7] S. F. Edwards and R. B. S. Oakeshott, Physica A 157, 1080 (1989).

[8] E. R. Nowak, J. B. Knight, M. Povinelli, H. M. Jaeger, and S. R. Nagel, Powder Technology 94, 79 (1997).

[9] J. B. Knight, C. G. Fandrich, C. N. Lau, H. M. Jaeger, and S. R. Nagel, Physical Review E 51, 3957 (1995).

[10] E. R. Nowak, J. B. Knight, E. Ben-Naim, H. M. Jaeger, and S. R. Nagel, Physical Review E 57, 1971 (1998).

[11] T. Boutreux and P. G. de Gennes, Physica A 244, 59 (1997).

[12] E. Caglioti, V. Loreto, H. J. Herrmann, and M. Nicodemi, Physical Review Letters 79, 1575 (1997).

[13] K. L. Gavrilov, Phys. Rev. E 58, 2107 (1998).

[14] D. A. Head and G. J. Rogers, J. Phys. A 31, 107 (1998).

[15] S. J. Linz, in Perspective look at nonlinear media. From physics to biology and social sciences, edited by J. Parisi, S. C. Muller and W. Zimmermann (Springer-Verlag, Berlin, 1998), p. 110.

[16] E. Ben-Naim, J. B. Knight, E. R. Nowak, H. M. Jaeger, and S. R. Nagel, Physica D 123, 380 (1998).

[17] G. C. Barker and A. Mehta, Physical Review E 40, 184 (1993).

[18] A. Coniglio and H. J. Herrmann, Physica A 225, 1 (1996).

[19] M. Nicodemi, A. Coniglio, and H. J. Herrmann, Physical Review E 55, 3962 (1997).

[20] A. Kolan, E. R. Nowak, and A. V. Tkachenko, Physical Review E 59, 3094 (1999).

[21] P. M. Chaikin and T. C. Lubensky, Principles of Condensed Matter Physics (University Press, Cambridge, 1995).
[22] Z. Dogic and S. Fraden, Physical Review Letters 78, 2417 (1997).

[23] A. Ciferri, Liquid Crystallinity in Polymers (VCH, New York, 1991).

[24] L. Onsager, Ann. (N.Y.) Acad. Sci. 51, 627 (1949).

[25] V. A. Baulin and A. R. Khokhlov, Physical Review E 60, 2973 (1999).

[26] C. C. Mounfield and S. F. Edwards, Physica A 210, 279 (1994).

[27] R. J. Buchalter and R. M. Bradley, Physical Review A 46, 3046 (1992).

[28] B. J. Buchalter and R. M. Bradley, Europhysics Letters 26, 159 (1994).

[29] B. S. Neumann, in Flow Properties of Disperse Systems, edited by J. J. Hermans (North-Holland, Amsterdam, 1953), p. 382.

[30] E. R. Nowak, J. B. Knight, E. Ben-Naim, M. Povinelli, H. M. Jaeger, and S. R. Nagel, in Powders and Grains '9'7 (Third International Conference on Powders and Grains, Durham, NC), edited by R. P. Behringer and J. T. Jenkins (Balkema, Rotterdam, 1997), p. 377.

[31] Recent experiments on sphere packings show that the system responds to changes in $\Gamma$ differently on very short, very long and intermediate time scales. On very short time scales or in the final steady-state, a reduction in $\Gamma$ leads to an increase in $\rho$, while an increase produces larger voids and thus a smaller $\rho$. The irreversible behavior occurs over intermediate length tapping intervals (C. Josserand, A. Tkachenko, D. M. Mueth and H. M. Jaeger, preprint).

[32] D. Bideau, A. Gervois, L. Oger, and J. P. Troadec, J. Physique 47, 1697 (1986).

[33] T. A. J. Duke, G. C. Barker, and A. Mehta, Europhysics Letters 13, 19 (1990).

[34] D. C. Hong, S. Yue, J. K. Rudra, M. Y. Choi, and Y. W. Kim, Physical Review E 50, 4123 (1994).

[35] M. Nicodemi, A. Coniglio, and H. J. Herrmann, Physica A 240, 405 (preprint).

[36] M. Adams, Z. Dogic, S. L. Keller, and S. Fraden, Nature 393, 349 (1998).

[37] S. F. Edwards and C. C. Mounfield, Physica A 210, 290 (1994). 